\title{
A delayed diagnosis of Blue rubber bleb nevus syndrome: characterized by refractory iron deficient anemia
}

\author{
Qiaoli Li $^{1}$, Lele Zhang ${ }^{1}$, Liwei Fang ${ }^{1}$, Hong Pan ${ }^{1}$, Qian Liang ${ }^{1}$, Jingyu Zhao ${ }^{1}$, and Jun Shi $^{2}$ \\ ${ }^{1}$ Chinese Academy of Medical Sciences Institute of Hematology and Blood Diseases \\ Hospital \\ ${ }^{2}$ Affiliation not available
}

February 21, 2021

\begin{abstract}
Refractory IDA and chronic occult bleeding from GI tract could happen due to BRBNS. Clinicians should keep in mind to detect the underlying diseases from GI tract by endoscopy. Precision diagnosis and appropriate intervention could booster to upgrade the quality of life for patients.
\end{abstract}

A delayed diagnosis of Blue rubber bleb nevus syndrome: characterized by refractory iron deficient anemia Running head: A case of Blue rubber bleb nevus syndrome

Type of manuscript: Case report

Qiaoli Li ${ }^{1 \#}$, Lele Zhang ${ }^{1 \#}$, Liwei Fang ${ }^{1 *}$, Hong Pan ${ }^{1}$, Qian Liang ${ }^{1}$, Jingyu Zhao ${ }^{1}$, Jun Shi $^{1}$

${ }^{1}$ State Key Laboratory of Experimental Hematology, National Clinical Research Center for Blood Diseases, Regenerative Medicine Clinic, Institute of Hematology \& Blood Diseases Hospital, Chinese Academy of Medical Sciences \& Peking Union Medical College, Tianjin 300020, China

\#These authors contributed equally

*Correspondence: Liwei Fang,fangliwei@ihcams.ac.cn

Abstract: Blue nevus syndrome (BRBNS) is a rare congenital disease characterized by multifocal venous malformations with involvement of skin and gastrointestinal (GI) tract. Here we represent a case with delayed diagnosis of BRBNS after treatment of refractory iron deficient anemia (IDA) for 12 years. The underlying disease was confirmed by GI endoscopy and capsule endoscopy. To obtain long-term curation, this patient received sigmoid-colectomy to remove the gastrointestinal lesions, and the level of hemoglobin maintained normal after surgery. In conclusion, BRBNS should be considered for patients with refractory IDA, and appropriate intervention could improve the level of hemoglobin and the quality of life.

Key words: Blue rubber bleb nevus syndrome; refractory iron deficient anemia; sigmoid-colectomy

\section{Introduction}

Blue rubber bleb nevus syndrome (BRBNS) is a rare congenital disease characterized by multifocal venous malformations, mostly involved in skin and gastrointestinal (GI) tract ${ }^{[1]}$. The positon of venous malformations can run through the GI tract, and the small intestine is the most common lesion location, which cause chronic occult bleeding and iron deficient anemia (IDA). The diagnosis of BRBNS is developed when typical malformations of venous appears ${ }^{[2]}$, but some cases had underground lesions leading to long term 
misdiagnosis. Here, we report a case misdiagnosed with refractory IDA for 12 years, who ultimately display the underlying disease of BRBNs uncovered by GI endoscopy and capsule endoscopy.

\section{Case presentation}

A 25-year-old female with a complaint of discontinuous pallor and fatigue for 12 years was admitted to the hospital. She received intermittent blood transfusion due to refractory anemia. Melena, hematemesis, menorrhagia or any other blood-loss events were denied. She showed a height of $145 \mathrm{~cm}$ and a weight of 43 $\mathrm{kg}$, mild pallor was noted and two small cutaneous bluish nodules were found in limbs. A series of laboratory findings demonstrated severe microcytic hypochromic anemia (hemoglobin $49 \mathrm{~g} / \mathrm{L}$, mean corpuscular volume $70.5 \mathrm{fL}$, mean corpuscular hemoglobin $18.5 \mathrm{pg}$, mean corpuscular hemoglobin concentration $263 \mathrm{~g} / \mathrm{L}$ ). Serum iron was $1.76 \mathrm{umol} / \mathrm{L}$ and total iron binding capacity was $94.25 \mathrm{umol} / \mathrm{L}$. Both bone marrow smear and biopsy revealed reduction of iron staining and the chromosome karyotype was 46, XX. Fecal occult blood test (FOBT) were negative for three times. Neither liver nor renal dysfunction was found. Colonoscopy indicated a sessile polyp measuring approximately $1.8^{*} 1.8 \mathrm{~cm}$ located in ascending colon about $60 \mathrm{~cm}$ from the anus, which was rough and bluish (Fig. 1A). At a distance of $30 \mathrm{~cm}$ from the edge of the anus, the sigmoid colon was hyperemic and congested with mucosal erosions and ulcers (Fig. 1B), the rectum showed blue mucosa with multiple site erosions located at the $10 \mathrm{~cm}$ from the anus (Fig. 1C). Capsule endoscopy showed the small intestine mucosa was blue and the blood vessels were large and tortuous (Fig. 1D-1E). Hence, she was diagnosed as BRBNS.

To improve the level of anemia, we administered 4 units of packed red blood cells transfusion and intravenous infusion of $200 \mathrm{mg}$ iron sucrose three times a week regularly. What is expected, we observed a significant increase of hemoglobin. The levels of hemoglobin rise from $56 \mathrm{~g} / \mathrm{L}$ to $89 \mathrm{~g} / \mathrm{L}$ in two weeks (Fig. 2A). To obtain long-term curation, she received a sigmoid-colectomy to remove the gastrointestinal lesions. Multiple purple lesions of sigmoid colon and rectum were showed by the using of laparoscope, and hemangioma was identified by histologic features. All the data were consistent with the diagnosis of BRBNs. The hemoglobin was maintained at the normal level after surgery (Fig. 2B).

\section{Discussion}

As a rare disease, the clinical manifestations of BRBNS is varied dependent on organs involvement. The lesions often occur at the cutaneous and GI tract. The typical lesions of cutaneous are only a few or quite a few scattered dark blue nevus with different size, usually asymptomatic [3]. The notable symptoms with GI tract lesions are hemorrhage and secondary anemia due to digestive tract blood loss ${ }^{[4]}$. A series of reports applause that video capsule endoscopy can provide direct and complete scanning of small-bowel lesions [2]. For this patient, she has suffered refractory IDA for 12 years, without any obvious signs of GI tract hemorrhage including hematochezia, melena, and negative FOBT many times. Misdiagnosis and delayed curation often occurs in such patients without significant cutaneous lesions or GI tract bleeding events.

The etiology of BRBNS is still unknown, and the majority of cases are sporadic without definite family history. It has been described an autosomal dominant transmission in some cases, with the responsible locus in 9p chromosome ${ }^{[5]}$. Recently, Soblet et al. discovered that somatic mutations in TEK (the gene encoding TIE2) could result in ligand-independent activation of TIE2 and lead to BRBNS ${ }^{[6]}$. The treatment of BRBNS includes supportive therapy ${ }^{[2]}$, pharmacological interventions ${ }^{[7]}$, and endoscopic treatments ${ }^{[8]}$. Surgery is reserved for patients with significant hemorrhage or other complications ${ }^{[9]}$. Nowadays, some researchers also propose that sirolimus is useful for the patients with BRBNS. However, there are no clear guidelines regarding dosing regimen, and the long-term safety ${ }^{[10]}$.

Until now, there is no available guidelines for diagnosis and management for BRBNS. The management of BRBNS generally depends on the extent of intestinal involvement and presence of other organ involvement, we just simply outline the management and treatment of BRBNS (Fig. 3). If patients only exhibit intermittent occult bleeding or mild anemia, palliative options such as iron supplementation and blood transfusion are usually chosen ${ }^{[2]}$. When patients have massive hemorrhage, refractory severe anemia, or related GI complications such as intussusception, rupture, gangrene, volvulus, and infarction, surgery or endoscopic 
treatment are recommended ${ }^{[11]}$. The successful use of sirolimus was recently reported in the treatment of BRBNS ${ }^{[12]}$. However, sirolimus has potential side effects on renal function, bone marrow, and cholesterol metabolism. In consideration of the patient's young age, we did not adopt this method. In this patient, we first deliver conservative therapies to improve the severity of anemia, and then we perform surgical resection successfully. She is in the remission condition but further follow-up is still needed.

Refractory IDA and chronic occult bleeding from GI tract could happen due to BRBNS. Clinicians should keep in mind to detect the underlying diseases from GI tract by endoscopy. Precision diagnosis and appropriate intervention could booster to upgrade the quality of life for patients.
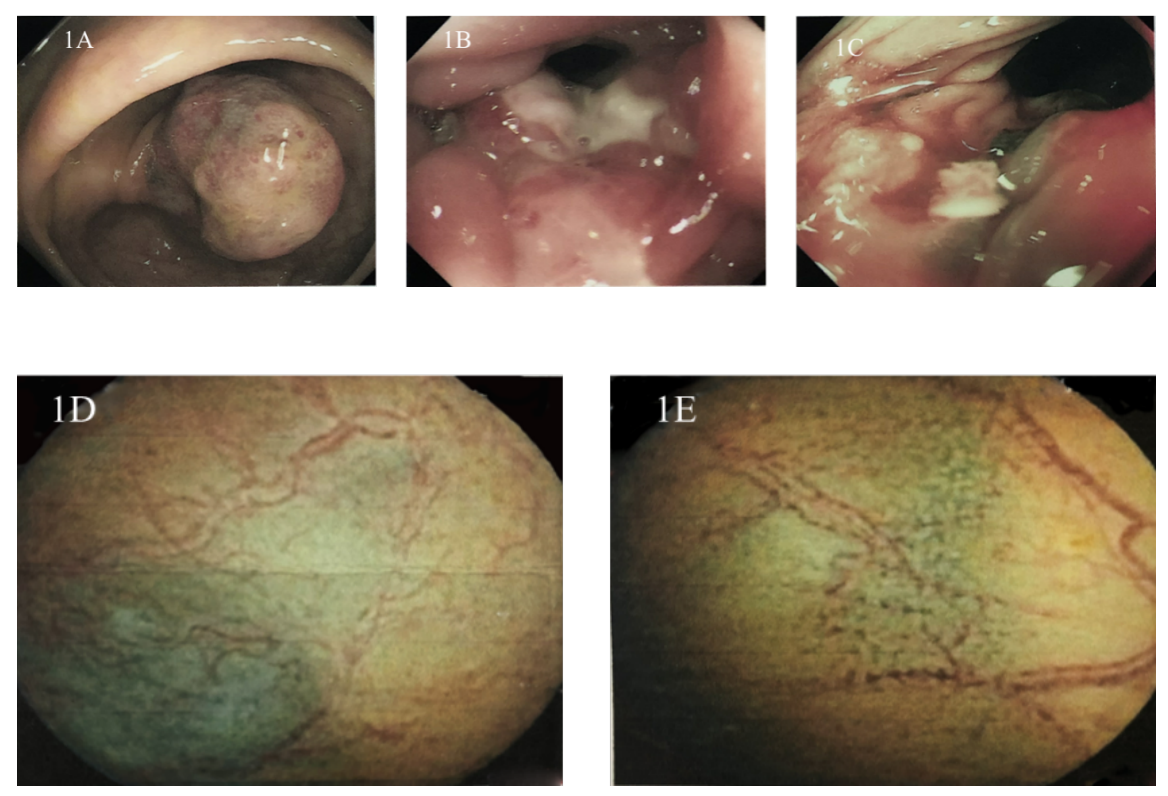

Figure 1. Images of colonoscopy (A-C) and capsule endoscopy (D-E). Rough bluish mass of the ascending colon (A); Erosions and ulcers of the sigmoid colon (B); Blue mucosa and multiple erosions in rectum (C); Bluish mucosa in small intestine (D); Tortuous vessels in small intestine (E)
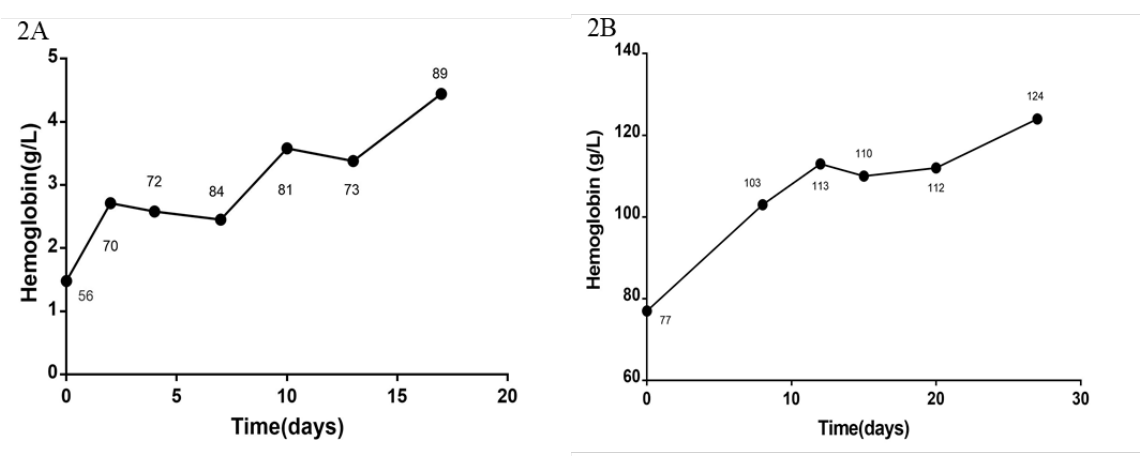

Figure 2. The improvement of hemoglobin after red blood cell transfusion and iron supplementation (A), and sigmoid-colectomy by laparoscopic (B). 


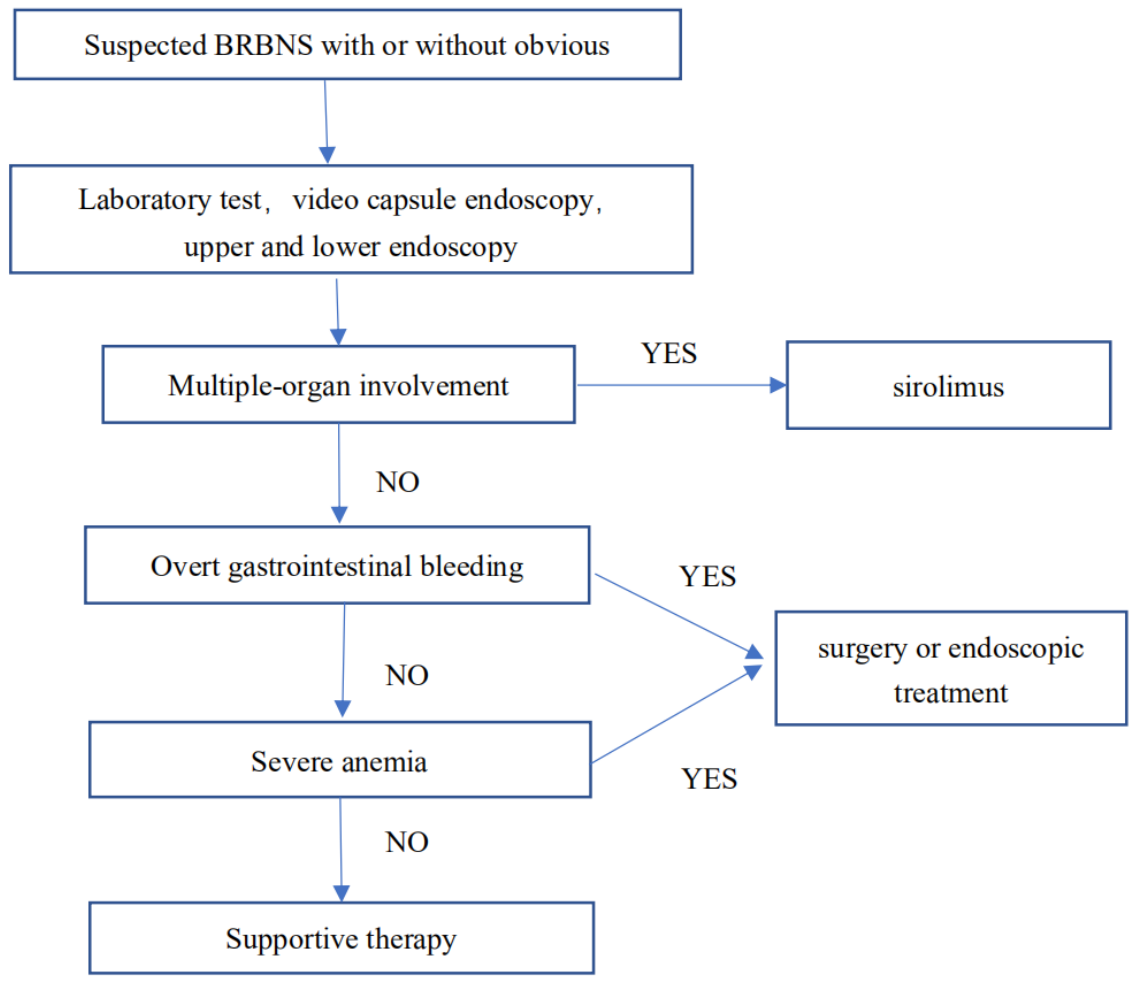

Figure 3. Diagnosis and treatment roadmap of BRBNS.

Conflict of interest: The authors declare that they have no conflict of interest.

A summary of relevant information will be published with the manuscript.

\section{References}

[1] Martinez CA, Rodrigues MR, Sato DT, Silveira Junior PP, Gama RF, Mattavelli CB, et al. Blue rubber bleb nevus syndrome as a cause of lower digestive bleeding. CaseRep Surg. 2014;2014:6834.https://doi.org/10.1111/j.1651-2227.2009.01608.x

[2] Agnese M, Cipolletta L, Bianco MA, Quitadamo P, Miele E, Staiano A. Blue rubber bleb nevus syndrome. Acta Paediatr. 2010;99(4):632-5.https://doi.org/10.1007/s12262-017-1715-y

[3] Menegozzo CAM, Novo F, Mori ND, Bernini CO, Utiyama EM. Postoperative dissem-inated intravascular coagulation in a pregnant patient with Blue Rubber Bleb Nevus Syndrome presenting with acute intestinal obstruction: Case report and literature review. Int J Surg Case Rep. 2017;39:2358.https://doi.org/10.1111/ped.12929

[4] Doi T, Masumoto N, Sonoda M, Nakayama H, Mizuno Y. Blue rubber bleb nevus syndrome with knee joint disorder. Pediatr Int. 2016;58(8):740-3.https://doi.org/10.17235/reed.2019.6250/2019.

[5] Unlusoy Aksu A, Sari S, Egritas Gurkan O, Dalgic B. Favorable Response to Sirolim-us in a Child With Blue Rubber Bleb Nevus Syndrome in the Gastrointestinal Tract. J Pediatr Hematol Oncol. 2017;39(2):1479.https://doi.org/10.3748/wjg.v20.i45.17254.

[6] Soblet J, Kangas J, Nätynki M, Mendola A, Helaers R, Uebelhoer M, et al. Blue Ru-bber Bleb Nevus (BRBN) Syndrome Is Caused by Somatic TEK (TIE2) Mutations. J Invest Dermatol. 2017;137(1):20716.https://doi.org/10.1155/2014/683684. 
[7] Yuksekkaya H, Ozbek O, Keser M, Toy H. Blue rubber bleb nevus syndrome: successful treatment with sirolimus. Pediatrics 2012;129(4):e1080-4.https://doi.org/10.1016/j.ijscr.2017.08.026.

[8] Ning S, Zhang Y, Zu Z, Mao X, Mao G. Enteroscopic sclerotherapy in blue rubber bleb nevus syndrome. Pak J Med Sci. 2015;31(1):226-8.https://doi.org/10.12669/pjms.311.5858.

[9] Aravindan U, Ganesan R, Thamarai Kannan M. Surgery for Blue Rubber Bleb Nevus Syndrome-a Case Report. Indian J Surg. 2018;80(3):272-4.

https://doi.org/10.1155/2018/7654278.

[10] Fernandez Gil M, Lopez Serrano P, Garcia Garcia E. Successful management of ane-mia with sirolimus in blue rubber bleb nevus syndrome: case report and update. RevEsp Enferm Dig. 2019;111(8):643647.https://doi.org/10.1016/j.jid.2016.0\%.034.

[11] Jin XL, Wang ZH, Xiao XB, Huang LS, Zhao XY. Blue rubber bleb nevus syndrom-e: a case report and literature review. World J Gastroenterol. 2014;20(45):172549.https://doi.org/10.1097/mph.0000000000000681.

[12] Ogu UO, Abusin G, Abu-Arja RF, Staber JM. Successful Management of Blue Rubb-er Bleb Nevus Syndrome (BRBNS) with Sirolimus. Case Rep Pediatr. 2018;2018:7654278.https://doi.org/10.1542/peds.20103611.
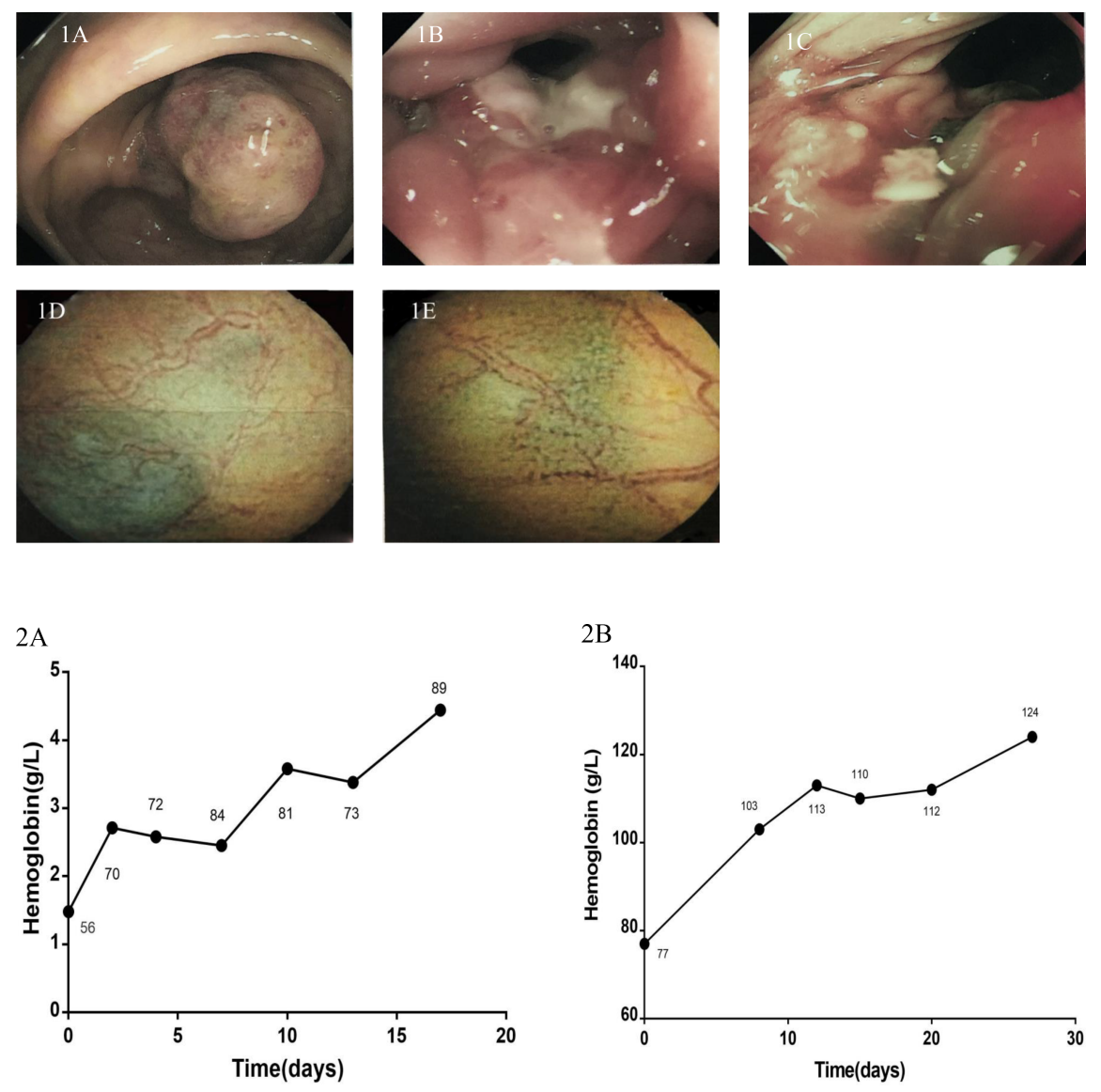


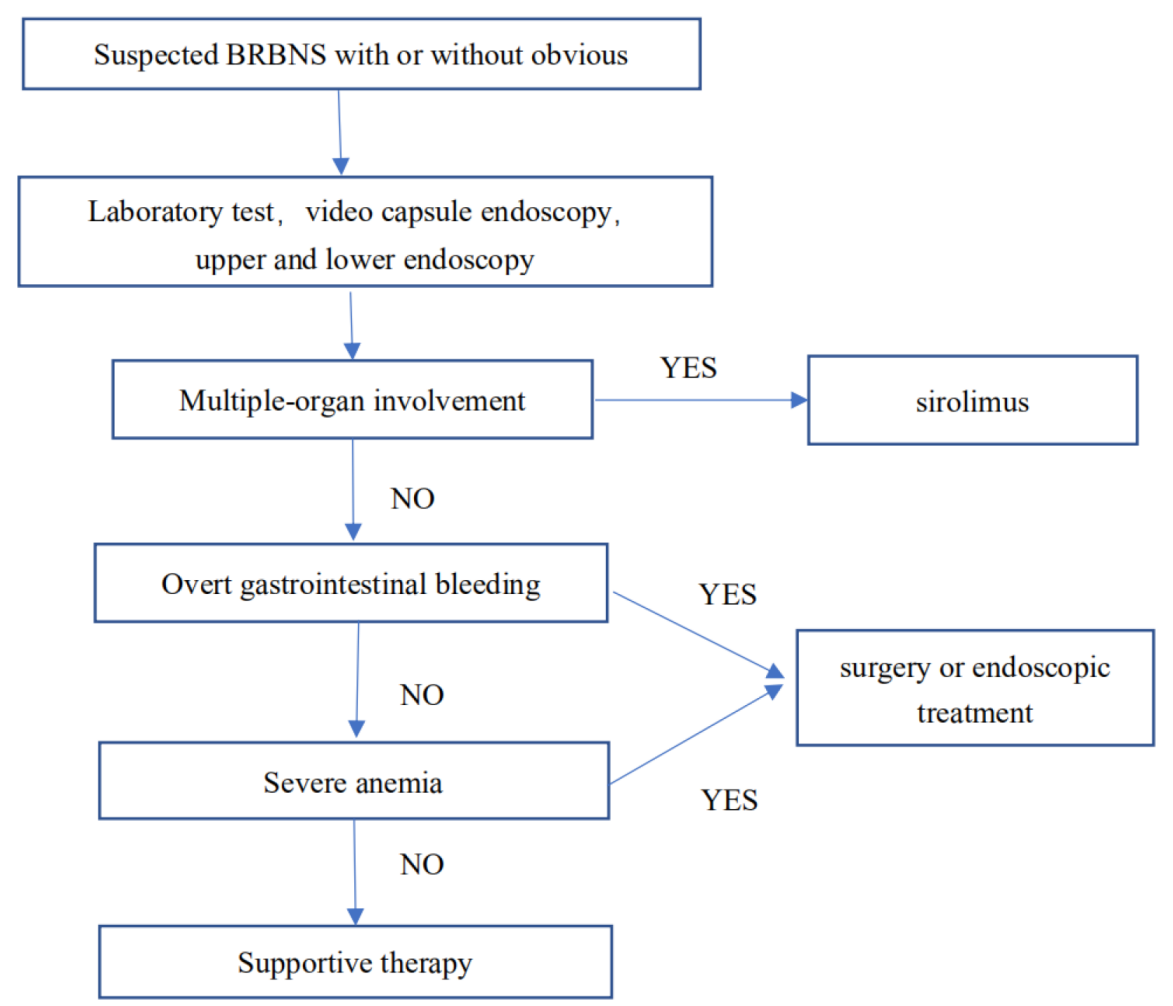

